\title{
Water, Man and Climate
}

\author{
Halidullin Oleg* \\ Ecology Professor, Academician of the Russian Federation LAN, Kazakh National University, Russia
}

Submission: March 12, 2017; Published: May 14, 2018

*Corresponding author: Halidullin Oleg, Ecology Professor, Academician of the Russian Federation LAN, Kazakh National University, Russia, Email: 7115215@mail.ru

\section{Opinion}

Water affects not only the environment, but also the climate. It is not just a working tool, a fluid reagent for transportation of heat, liquid, rotation of turbines of hydroelectric power stations, washing of objects and bodies. The water is not investigated. We use a small part of its physical properties. In nature, water, in contact with the shores, dissolves various substances and delivers them to their destination, providing underground and coastal living creatures and plants.

As a result of human activity, the functions of water have changed. We change the ways and purpose of water. In total, people took from nature $63 \%$ of the inhabited earth, each hectare of which contains 20 tons of underground living creatures. These are microbes, worms, etc. insects that, in symbiotic relationship with plants and terrestrial populations, absorb moisture, transform in food chains and economically exhale highly individual pairs, which we call natural or organic vapors. Global filtration is performed, which has been tuned for millions of years.

Human intervention in the water circuit destroys food chains, and water from asphalt and other destroyed areas evaporates immediately after precipitation. In essence, a person destroys a natural filter. Evaporation of waters that do not undergo biological filtration has a different molecular structure, another quality. These evaporation are artificial, unknown to nature. More artificial fumes are produced by industry and utilities around the world. Hardening, cooling, evaporation, each boiling kettle, every washed cup, car and human body takes to the sky a certain amount of water, not clean for nature, fumes. Such evaporation increases with the development of civilization, unprecedented volumes of water in the atmosphere grow, which have changed the established mechanism of atmospheric phenomena for centuries. The water cycle between the atmosphere and the soil has changed. The cyclicity, massiveness and ranges of precipitation have changed. Therefore, some areas suffer from floods, and others - from drought and fires. This is just the beginning of the finals - a general flood.

Are there real opportunities for reducing natural disasters, stopping climate change?

Under the Paris Agreement, all countries pledged to reduce emissions of carbon dioxide. We decided that it was all about greenhouse gases. But this is a mistake. Moreover, this error leads the planet to a catastrophe, distracting mankind for microscopic effects on the climate. It is known that: "Since the beginning of the 20th century, according to UN experts, the increase in $\mathrm{CO}_{2}$ emissions was from 0.5 to $5 \%$ per year. As a result, over the past hundred years, 400 billion tons of carbon dioxide have been supplied to the atmosphere only by burning fuel."

Every year people irrevocably take from rivers and lakes about 2000 cubic km. Fresh water, which is about 5\% of the world's rivers flow. Annually, all this water goes back to the sky with structural changes of a different type. They are alien to nature.

In the $1^{\text {st }}$ cubic $\mathrm{km}$ there are $1000 \times 1000 \times 1000=1000000000$ or 1 billion cubic m. Multiply by 2000 and get 2 trillion cubic meters. m. or 2 tr. tons of water. All this water through direct evaporation and evaporation from the sewage sump goes into the atmosphere, not fulfilling its historical mission. Hence it follows that a new balance has emerged in the single circulation of water. All this water rises into the atmosphere. Annually 2000 cubic meters, kilometers. Therefore, 400 billion tons of $\mathrm{CO}_{2}$ in 100 years or 4 billion tons per year is 0.2 percent of the water vapor. It should be added to this the volumes of natural evaporation water from the remaining biota, it remained 100 $63=37 \%$ of the inhabited land. Perhaps this percentage is getting even smaller. As confirmed by reference data: “The Earth's atmosphere is to a large extent a product of living organisms. The approximate composition of the Earth's atmosphere: $78.08 \%$ nitrogen, $20.95 \%$ oxygen, a variable amount of water vapor (average about 1\%), 0.93\% argon, $0.038 \%$ carbon dioxide, and a small amount of hydrogen, helium, other noble gases and pollutants ". This is "an average of about 1\%" - a long-obsolete figure and needs clarification. According to the carbon dioxide gas, it was calculated and determined: from 0,038 to $0,2 \%$. The same research is required on the evaporation of water in two types of organic and artificial.

\section{Conclusion}

Not carbon dioxide, and evaporation of water is the main cause of natural disasters and climate change.

The purpose of this article is to attract the attention of the academic world to create a new movement to prove the 
Hypothesis. This will create a new unified concept for the preservation of life on the planet. The basis of this concept should be the all-round return to nature of organic fumes and the reduction of artificial fumes. To do this, all h Humanity must pass to total water saving in industry, communal services, in everyday life by every continent, every state, and every person.

The main elements are:

a) Complete and unconditional stoppage of projects and construction of new reservoirs. Issue of old reservoirs with reclamation of flooded areas. Several hundred new reservoirs are being commissioned annually on the globe. The total area of all the reservoirs of the world is more than 600 thousand km2. Many of the world's rivers - the Volga, the Dnieper, the Angara, Missouri, Colorado, Parana and others have been turned into cascades of reservoirs.

b) Revision of all farming with the introduction of nondiscarded plowing, drip irrigation. Elimination of irrigation and irrigation systems and canals, rice checks. Example: Israel, which, using drip irrigation, exports its plant products

c) Ensure the utilization or recycling of waste with the cessation of contamination by landfills. Recultivation of existing garbage dumps and dumps of ore enterprises.
Scandinavian countries have already cleared all their territories of debris.

d) Strict water saving. It is necessary to create a new ideology for the entire population of the Earth about total water saving. For example, stop squandering water when washing. We brush our teeth and the water flows. Adjust the wash temperature, and clean water goes to the sewer. We wash away feces with clean drinking water, although it is known to use waste water after washing. We get water from water pipes, and from the roofs of the water we merge into the streets. Warm the water in one place, we spend in others. Heat the pipes, atmosphere and mix with cold in the shower, near the sink. Known and widely used devices for economical water consumption in airplanes, trains

e) Landscaping of roofs and walls of all buildings and structures. To create a new direction - growing on them greens, vegetables and fruits. There are houses with vertical landscaping

f) Transfer of all types of production and urban infrastructure under the ground and under water, gradually and housing. Today, a significant part of the population moves to the metro, lives and works in underground enterprises. Japan is building an underwater house - a city.

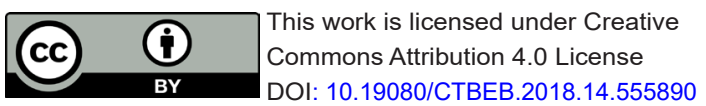

